\title{
Troppo poca DP in Italia
}

\author{
Giusto Viglino
}

\author{
Unità Operativa Aziendale di Nefrologia e Dialisi \\ Ospedale San Lazzaro, Alba - Cuneo
}

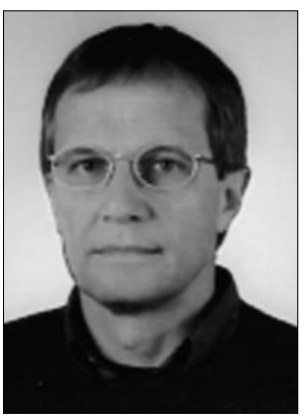

Oltre 20 anni dalla riedizione della dialisi peritoneale (DP) con la tecnica della CAPD, questa metodica rappresenta poco più del $10 \%$ dei pazienti in trattamento sostitutivo in Italia. Tale percentuale è tra le più basse dei Paesi sviluppati e colloca di fatto la DP come un trattamento marginale dell'uremia. Pertanto sorge spontaneo chiedersi se tale bassa penetrazione sia da imputare a una scarsa efficacia della metodica o ad altre ragioni.

Nel corso di questi anni in Italia è stato raggiunto il pieno trattamento sostitutivo dell'insufficienza renale cronica senza un sostanziale problema di limitazione delle risorse destinate a questa terapia e quindi senza nessuna spinta economica a diffondere trattamenti a più basso costo come la DP. Uno scenario diverso potrebbe verificarsi nel momento in cui il Servizio Sanitario Nazionale non provvederà più ad appianare il deficit delle strutture sanitarie.

Tuttavia la penetrazione della DP risulta disomogenea da Regione a Regione toccando le punte più basse in quelle in cui vi è stata una forte presenza dell'emodialisi (ED) privata parallelamente a una scarsità di posti dialisi nel settore pubblico. Le limitazioni legali legate all'accreditamento per effettuare la EP nelle strutture private e/o la maggiore remuneratività dell'ED sono i fattori che principalmente impediscono la penetrazione della DP in queste $\mathrm{Re}$ gioni.

D'altra parte la disomogeneità della prevalenza della DP anche tra le Regioni in cui non si è verificata una concorrenza del settore privato deve trovare spiegazione in altre ragioni. Tra queste molto probabilmente la principale è legata alla convinzione che la DP non rappresenta una reale opzione terapeutica del trattamento sostitutivo ma un trattamento di seconda scelta da utilizzarsi solo quando vi siano ostacoli clinici e soprattutto organizzativi a effettuare l'ED (risorse limitate, carenza di posti in ED, difficoltà di trasporto del paziente al Centro dialisi). Questa convinzione, ormai sconfessata da molti dati della letteratura, fa in modo che non vengano nemmeno ricercate quelle condizioni cliniche, sociali, psicologiche e attitudinali che possono trarre beneficio dall'utilizzo della DP come prima opzione terapeutica. D'altra parte la maggiore necessità di risorse in termini di personale, attrez- zature e locali che occorre investire per un programma di ED rispetto a uno di DP, conferisce a chi lo gestisce un maggiore potere contrattuale e una maggiore visibilità nei confronti del sistema sanitario.

La carenza di riferimenti legislativi relativi al personale, ai locali e alle attrezzature da utilizzare in DP così come i rimborsi tariffari spesso meno remunerativi per la DP rispetto all'ED, non sono altro che lo specchio di quanto poca attenzione sia stata riservata alla DP nel corso di questi anni nel nostro Paese. Tali rimborsi risultano ancora meno adeguati quando sono rapportati all'utilizzo delle nuove soluzioni dialitiche quali il bicarbonato e le icodestrine, o all'uso dell' APD. Questo limita la possibilità di ottenere risorse per la DP nell'ambito della normale dialettica contrattuale all'interno dell'Unità Operativa di appartenenza o nei confronti dei responsabili amministrativi della struttura sanitaria. Di conseguenza la DP è stata effettuata nei diversi Centri in modo disomogeneo e spesso con risorse assolutamente insufficienti. Pertanto sarebbe opportuno chiedersi se la DP non abbia dato molto di più di quello che ha ricevuto e cosa avrebbe potuto dare in termini di risultati se avesse proporzionalmente avuto le stesse risorse riconosciute all'ED. 
Un altro elemento che può avere influito sulla diffusione della DP è legato al fatto che l'organizzazione della rete dei Centri dialisi sul territorio è stata dimensionata per garantire una efficienza operativa basata sulle necessità dell'ED. Un incremento della DP a valori del $25-30 \%$ può porre dei problemi sulla utilizzazione dei posti letto esistenti di ED, anche se l'atteso incremento della popolazione dialitica potrebbe ridimensionare tale problema. D'altra parte, con gli attuali livelli di penetrazione della DP, nei Centri dialisi di piccole dimensioni è difficile raggiungere un numero di pazienti tali da ammortizzare le risorse, soprattutto umane, necessarie per un buon funzionamento di un programma di DP. Infatti l'incidenza di peritoniti e il drop-out della tecnica sono condizionate dal numero di pazienti in trattamento e dalla esperienza acquisita dal Centro; quest'ultima raggiungibile disponendo di un medico responsabile e di infermieri dedicati alla DP. Tuttavia l'autonomizzazione del personale infermieristico richiede almeno 3 unità in modo da far fronte alle necessità di riposi, ferie, turni di reperibilità e alla possibilità di malattia o di rotazione del personale. Tale impiego di risorse può essere adeguatamente ammortizzato con almeno $15-20$ pazienti stabilmente in trattamento e coinvolgendo il personale medico e infermieristico nel protocollo di scelta del trattamento sostitutivo che dovrebbe essere realizzato nei mesi precedenti l'inizio del trattamento dialitico.

Con tale protocollo è possibile provvedere oltre che alla valutazione clinica dei pazienti anche all'informazione adeguata dei pazienti e dei familiari sulla possibilità di un trattamento dialitico domiciliare autogestito, valutandone la fattibilità e l'accettabilità sia dal punto di vista sociale, attitudinale e psicologico. Questo obiettivo può essere facilmente raggiunto se durante il predialisi si associano alle tradizionali visite ambulatoriali, incontri di gruppo tra l'équipe curante e i pazienti, un colloquio del paziente con lo psicologo, una visita dell'infermiere al domicilio del pa- ziente. L'assenza di tale protocollo condiziona negativamente la diffusione della DP. È stato infatti dimostrato come una scarsa informazione, oltre a essere una procedura deontologicamente scorretta, determini anche percentualmente una minore scelta della DP. Inoltre solo nel 25$30 \%$ dei casi la scelta del trattamento è su base clinica mentre nella restante parte dei casi è determinata da fattori sociali, psicologici e attitudinali che devono essere adeguatamente valutati. Tra questi, per la scelta della DP, sicuramente rilevante è l'idoneità dell'ambiente fisico e familiare in cui si dovrà svolgere il trattamento dialitico, la motivazione e la capacità all'autogestione della terapia. D'altra parte una mancata valutazione di tali fattori è sicuramente in grado di determinare un incremento delle principali cause di drop-out dalla DP, quali la peritonite, la terapia inadeguata da scarsa compliance alla prescrizione dialitica, il burn-out del paziente e/o dei familiari.

A questo riguardo occorre ricordare che oltre il $40 \%$ dei pazienti in DP effettua il trattamento con l'aiuto di un partner e che questa percentuale incrementa negli anziani e nei diabetici. Dal momento che in dialisi tende progressivamente a aumentare il numero di pazienti anziani che vivono da soli o non autosufficienti, la possibilità di disporre in futuro di modelli organizzativi che consentano di assistere i pazienti in DP a domicilio o in RSA, sarà un fattore che potrà condizionare la diffusione di questo trattamento dialitico. Pertanto è prospettabile un modello organizzativo a quattro gradini che al primo posto prevede la dialisi autogestita, al secondo una gestione del trattamento da parte di un partner consanguineo o non, al terzo e quarto gradino la dialisi gestita da personale sanitario a domicilio $o$ in RSA. Mentre per i primi due livelli la scelta del trattamento può cadere sia sulla CAPD che sull'APD, per gli ultimi due gradini gli aspetti organizzativi privilegiano l'APD.

In conclusione ci sembra di poter affermare che la bassa diffusione della DP in Italia non sia da attribuire a una scarsa efficacia della metodica che, se correttamente applicata, è in grado di fornire risultati sovrapponibili a quelli dell'ED, ma a scelte clinico-organizzative spesso basate su dei pregiudizi e/o su valutazioni economiche.

nefrologia@asl18.sanitacn.it 